\title{
Endocarditis from Klebsiella Pnumoniae producing Carbapenemase, an Emerging
} infection?

\author{
Albanese $\mathrm{A}^{{ }^{1 *}}$, Scordo $\mathrm{P}^{1}$, Smedile $\mathrm{V}^{1}$, Patanè $\mathrm{F}^{\mathbf{2}}$ \\ ${ }^{1}$ UOSD Infectious Diseases A.O. Papardo, Messina, Italy \\ ${ }^{2}$ UOC Heartsurgery A.O. Papardo Messina, Italy
}

*Corresponding author: Albanese A, UOSD Infectious Diseases A.O. Papardo, Messina, Italy.

Received date: December 20, 2019; Accepted date: January 01, 2020; Published date: January 06, 2020

Citation: Albanese A., Scordo P., Smedile V., Patanè F. (2020) Endocarditis from klebsiella pnumoniae producing carbapenemase, an emerging infection?. J Clinical Research and Reports, 1(1); DOI:10.31579/jcrr/2020/012

Copyright: () 2020 Albanese A, This is an open access article distributed under the Creative Commons Attribution License, which permits unrestricted use, distribution, and reproduction in any medium, provided the original work is properly cited.

Abstract
In the literature there are still few cases of endocarditis caused by non-HACEK gram negative bacteria,
particularly by Klebsiella pneumoniae carbapenemasi producing [2,3].
The few cases reported mostly concern patients who were already hospitalized for other causes [1,2,3]. In this
article we report two cases of endocarditis arising in non-hospitalized patients. One of the two patients died
decayed.
The death due to the consequences of the ischemic instinct that was the cause of the hospitalization.
The other patient was subjected to aortic valve replacement and you have subsequent checks showing no signs
of infections and the valve works regularly.
Both cases were treated with ceftazidime avibactam plus gentamicin.
Key words: Endocarditis

Introduction

Endocarditis from non-HACEK gram negative bacteria are very rare, ranging between 1 and $3 \%$ of endocarditic infections [1, 2]. The majority of cases concern patients who have undergone numerous hospital visits, which are carriers of cardiac devices (for example, a pacemaker) $(4,5)$.

The mortality rate of this type of infection is around, according to data from the International Collaboration Study on Endocarditis Prospective Cohort Study and the Italian Endocarditis Study (ICEPCS) [1], around $25 \%$.

The mortality rate increases if MDR bacteria are isolated.

In this paper we report two cases of patients who entered the hospital for non-traumatic reasons and who in their clinical history did not report hospital admissions or access to health facilities for chronic diseases.

\section{Case report:}

\section{CASE 1}

The first case concerns a young patient who accesses the emergency room for loss of conscience preceded by the phase of dysarthria and difficulty in moving.

The patient was subjected to brain CT and subsequent magnetic resonance imaging that showed large ischemic area in the bilaterally occipital region and thalamic.

In the anamnesis, the relatives, did not report particular pathologies neither accesses to sanitary structures.

The patient was therefore subjected to an echocardiographic examination that showed the presence of vegetation on the native mitral valve. Blood collection samples were then performed and empiric antibiotic therapy was started with vancomycin, rifampicin and gentamicin, in accordance with the ESC 2015 guidelines [6].

Three days after execution, blood cultures were positive for klebsiella pneumoniae producing carbapenemase (KPC), with sensitivity only to gentamicin and colistin.

The patient who was in precarious clinical condition was evaluated by cardiac surgeons who excluded the possibility of valve prosthesis surgery.

Was therefore initiated, after doing also a research in the literature, antibiotic therapy with ceftazidime avibactam (CAZ-AVI) 2 gr / 500 $\mathrm{mg}$ i.v. three times a day and Gentamicin $80 \mathrm{mg} 3$ rows in $250 \mathrm{cc}$ of physiological sol infused in $30 \mathrm{~min}$. the patient was already under anticoagulants [2,5]. The treatment was prolonged for 4 weeks obtaining the negativization of the blood cultures, the negativization of the inflammation indexes and an almost total reabsorption of the endocarditic lesion.

The patient died due to brain damage two months after treatment. During this period did not show fever, blood cultures were kept negative as well as the indices of floogsi (C-reactive protein and Procalcitonin).

\section{CASE 2}

The second case concerns a 74-year-old man hospitalized, also under emergency conditions, for acute respiratory failure, without associated comorbidities. During the hospitalization in Reanimation, he underwent trans-esophageal echocardiography, which showed the presence of a severe aortic insufficiency with vegetation adherent to the valvular flaps. The patient performed blood cultures that were 
positive for KPC. after three days of hospitalization, the patient was estubied and in good compensation and had already started antibiotic therapy with AVI / CAZ 1,5 grx3 / die gentamicina $80 \mathrm{mg} 3$ vials in $250 \mathrm{cc}$ of saline solution in monosomministration daily, according to the antibiogram, was transferred to Caridosurgery where it was subjected to valve replacement surgery.

Continued antibiotic therapy for 4 weeks and to date all controls are negative, the patient is well and has resumed a normal life $[2,6]$

Discussion

Infectious endocarditis is a disease burned by high mortality and severe complications. Unfortunately, all the bacterial infections have the potential to involve the heart valves, making this diesease even more deadly [1]. In particular, in this age characterized by serious nosocomial infections caused by MDR and XDR microorganisms, this ability became especially dangerous, given the limited weapons we have in presence of such bacteria [7-9].

Endocarditis related to gram negative microorganisms not belonging to the HAECK group have been reported for more than 25 years, but their importance has increased during the last few years, when antimicrobial resistance became a world problem.

We presented two cases of endocarditis caused by KPC, both resolved thanks to the use of a "protected" cephalosporin: ceftazidime/avibactam. AVI/CAZ is one of the few weapons available for carbapenem resistant Enterobacteriacae. However, as expected, its use led to the appearance of AVI/CAZ resistant strain [10].

Therofore, ECDC issued an alert to prevent the spread of these strains, that suggest implementing measures of contact isolation whenever an AVI/CAZ resistant microorganism is found in a person, and to screen every admitted patient for potentially begin an asymptomatic carrier.

Moreover, the use of a treatment with AVI/CAZ during endocarditis caused by carbapenem resistant bacteria has been seldomly reported, and there are concerns about fact that such a prolonged therapy can determine a more rapid onset spread of resistance.

Conclusion:

Further studies are needed to determinate the association of prolonged therapy and the selection pressure towards the appearance of a resistance that would deprive us of the few antibiotics active in the case of carbapenem resistant microorganism.
Acknowledgment

We declare no conflict of interests.

References:

1. M. Falcone (2018) Risk factors and outcomes for endocarditis due to non-HACEK Gram-Negative Bacilli: Data from the prospective multicenter Italian endocarditis study cohort.

2. Iacovelli A (2018) A challenging case of carbapenemaseproducing Klebsiella pneumoniae septic thrombophlebitis and right mural endocarditis successfully treated with ceftazidime/avibactam. Infection 46(5): 721-724.

3. Angela Cano (2017) Risk of infection and mortality among patients colonized with Klebisella pneumoniae Carbapenemase-producing K. pneumoniae: Validation of scores and proposal for management. CID; 1-7.

4. M. Bassetti Multidrug resistant Klebisella pneumoniae: chellenges for treatment, prevention and infection. Expert review of anti-infective therapy.

5. ZR. Palacios-Baena (2017) Empiric therapy with carbapenem sparing regimens for bloodstream infections due to extended spectrum $\beta$ lactamase producting Enterobacteriaceae: results from the INCREMENT cohort. CID 2017, 65(10); 1615-23.

6. G. Habib (2015) ESC guidelines for the management of infective endocarditis of the European society of cardiology. European Herat J. advance access published august 29.

7. Mulani MS (2019) Emerging strategies to combact ESKAPE pathogens in the era of antimicrobial resistance: a review. Front Microbiol; 10:539.

8. Karaiskos I (2019) The "old" and the "new" antibiotics for MDR gram negative pathogens: for whom, when and how. Front Public Health; 7:151.

9. Brescini L (2019) Clinical and epidemiological characteristic of KPC-producing Klebsiella pneumoniae from bloodstream infections in a tertiary referral center in Italy. BMC Infect Dis; 19:611.

10. European Centre for Disease Prevention and Control. Emergence of resistance to ceftazidime-avibactam in carbapenem resistant Enterobacteriacae. European Centre for Disease Prevention and Control. 
This work is licensed under Creative Commons Attribution 4.0 License

To Submit Your Article Click Here: Submit Article

DOI:10.31579/jcrr/2020/012
Ready to submit your research? Choose Auctores and benefit from:

* fast, convenient online submission

* rigorous peer review by experienced research in your field

* rapid publication on acceptance

* authors retain copyrights

* unique DOI for all articles

* immediate, unrestricted online access

At Auctores, research is always in progress.

Learn more https://www.auctoresonline.org/journals/journal-of-clinicalresearch-and-reports 